\title{
Outcome Of Arthroscopic Vs Mini Open Subacromial Decompression In Treating Shoulder Impingement Syndrome
}

Nik Alyani Nik Abdul Adel ${ }^{1}$, Khairul Nizam bin Siron@Baharom ${ }^{1}$, Muhammad Taufik bin Mat Lani ${ }^{1}$

${ }^{1}$ Department of Orthopaedics, Traumatology and Rehabilitation, Kulliyyah of Medicine, International Islamic University Malaysia

Presenter: Nik Alyani Nik Abdul Adel

Introduction: Shoulder impingement syndrome is a common diagnosis leading to shoulder disabilities. Subacromial decompression surgery either arthroscopic or mini open is indicated in patients who failed conservative therapy. Arthroscopic surgery is introduced to reduce soft tissue dissection hence less soft tissue damage and fasten rehabilitation. This study is conducted to compare the outcome of mini open and arthroscopic surgery. Materials and Methods: A cross sectional study was conducted in patients who were diagnosed with shoulder impingement syndrome with or without rotator cuff tear who underwent subacromial decompression surgery, with mini open or arthroscopic surgery at Hospital Tengku Ampuan Afzan, Kuantan. Functional outcome was assessed using American Shoulder and Elbow Surgeon (ASES) score and Constant score. Results: Six patients were recruited in the mini open group while 11 in the arthroscopic group. Of these, all were female in mini open group, aged $56.7 \pm 7.9$ while seven female and four male in the arthroscopic group aged $55.1 \pm 9.4$ (p-value 0.733 ). They were evaluated at 3 to 5 years after surgery in the mini open, and at six months in the arthroscopic group. There was no significant different in the ASES score in the mini open group $(89.8 \pm$ $11.7)$ and arthroscopic group $(89.8 \pm 17.9)$, with p-value of 0.998 . Constant score in the mini open was $18.67 \pm 7.61$, while in the arthroscopic was $16.18 \pm 15.03$; and there was no significant difference in this two groups ( $p$-value 0.712 ). Three patients each has good and fair outcome in the mini open surgery, while six patients has excellent, two each has good and fair; and one has poor outcome in the arthroscopic surgery. Conclusion: There were no significant differences in the functional outcomes between mini open and arthroscopic subacromial decompression surgery. 